\title{
Stimulus-response compatibilities between vertically oriented stimuli and horizontally oriented responses: The effects of hand position and posture
}

\author{
CLAIRE F. MICHAELS and SUSAN SCHILDER \\ Lake Forest College, Lake Forest, Illinois
}

\begin{abstract}
Stimulus-response (S-R) compatibility effects between vertically oriented stimuli (above or below fixation) and horizontally oriented responses (left or right switch deflections by a single hand) have been shown to depend both on which hand responds (Bauer \& Miller, 1982) and on the location at which the response is made (eccentricity on a frontoparallel line; Michaels, 1989). In the latter study, hand position and hand posture were confounded, so it is unclear which variable determined the compatibility effect. In Experiment 1, the importance of effector position was tested. Vertically oriented stimuli were paired with a horizontal response solicited at different locations but always involving the same hand posture. Compatibility effects emerged, and their direction depended on position. In Experiment 2, the compatibilities were not evident in a simple reaction time paradigm, so the effect was not due to differential ease of responses. In Experiment 3, a change in hand posture (palm up or palm down) at the same location (the body midline) also affected the compatibilities. It was concluded that the S-R compatibility of orthogonally oriented stimuli and responses is influenced by (1) which hand responds, (2) the location of that hand, and (3) its posture. The results imply that both postural and positional states of the action system affect S-R compatibility.
\end{abstract}

In a recent paper, one of us (Michaels, 1988) has argued that the phenomenon of stimulus-response (S-R) compatibility might be explained with reference to the ecological approach to perception and action. An important principle in that approach is the mutual compatibility of perception and action; the information that modulates activity is itself modulated by the activity. Further, it is argued that both the nature and the state of the action system provide constraints on which stimulus patterns will regulate it.

That the nature of the action system conditions perception is illustrated by Warren's (1984) observation that leg length determines whether a stair will be perceived as climbable. That the state of the action system conditions perception is illustrated by Fitch and Turvey's (1978) interpretation of Hubbard and Seng's (1954) baseball batting analysis. The organization of the swing of a professional baseball batter into a step-swing couplet invites regulation by two types of information. The step portion is initiated by optical patterns that specify the pitcher's release of the ball, and the swing portion is initiated by optical patterns that specify time to contact. The organiza-

Experiments 1 and 2 were run as part of a senior honors thesis at Lake Forest College by Susan Schilder. The authors thank Larisa Stross and Megan Meschery for their assistance. They also thank Lester Krueger, Yosef Solomon, and two anonymous reviewers for their comments on an earlier draft. Correspondence and requests for reprints should be sent to Claire Michaels, Lake Forest College, Lake Forest, IL 60045. tional state of the batter's act determines the batter's informational needs.

The notion that states of the action system influence information (and, perforce, perception) has been suggested as a possible basis for S-R compatibility in the choice reaction time (RT) paradigm (Michaels, 1989). Such an interpretation was applied to Bauer and Miller's (1982) observation that during responses made with a left or right finger movement of the right hand to a stimulus above or below fixation, a "counterclockwise" pairing (see Figure 1) is faster than a "clockwise" pairing, but that during responses made with the left hand, the converse pairing is faster. The current state of the action systemthat is, which hand is the effector-determines the S-R compatibility (i.e., the faster and more accurate pairing).

However, if the responses are solicited at a location not on the body midline, the pairings that are compatible change. Michaels (1989) showed that when the effector is displaced $60 \mathrm{~cm}$ to the ipsilateral side of the body midline, the preferred pairing is opposite that revealed by Bauer and Miller (1982) for the body midline; the left hand is faster with counterclockwise pairing, and the right hand is faster with the clockwise pairing. It is critical to note that it is not the one or the other response that is faster, but rather both responses under a particular pairing rule; so an explanation of these phenomena in terms of the biomechanics of the responses is not sufficient.

Michaels (1989) concluded that the states of the action system that can determine S-R compatibility were, there- 


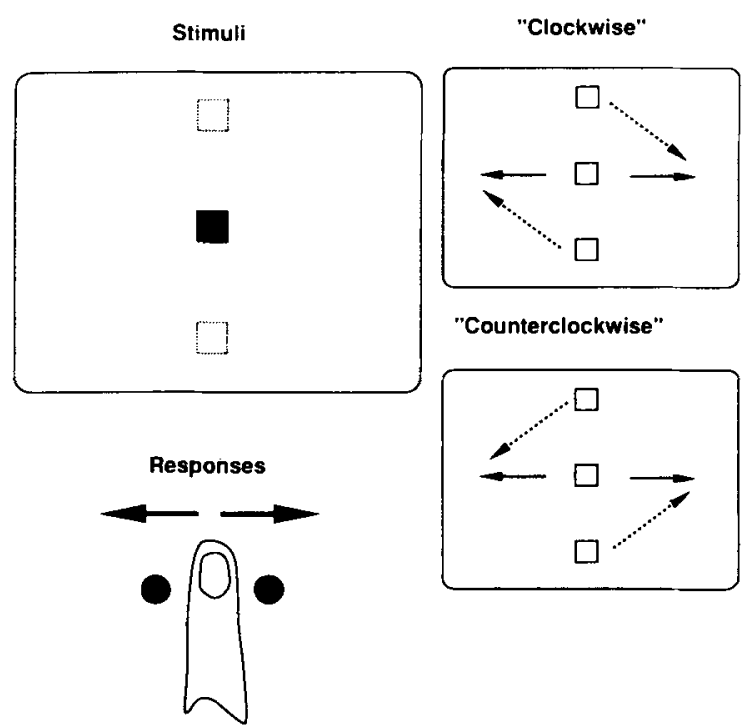

Figure 1. On the left is illustrated a computer screen with a darkened fixation square; above and below the square are the two locations at which the stimulus may appear. Beneath the screen is illustrated a finger that will respond to the left or right, depending on which stimulus appears. "Clockwise" and "counterclockwise" are shorthand for pairing rules that relate the stimuli above or below fixation to the responses to the left or right of a central position. In the illustration on the right, "clockwise" refers to aboveright/below-left and "counterclockwise" refers to above-left/belowright pairing rules. (Conversely, if the stimuli were horizontally disposed and responses vertically disposed, clockwise would refer to a left-up/right-down pairing.) Note, though, that responses are not rotations, but left or right movements.

fore, both the responding hand (as had been shown by Bauer \& Miller, 1982) and the response location. However, there is some ambiguity as to exactly what action-system variable constituted "response location." On the body midline, the subjects grasped the toggle switch with a palm-down (prone) hand; but at the eccentric position, this posture could not be adopted without uncomfortable wrist flexion, so the switch was grasped with a palm-inward (more supine) hand. It is unclear, therefore, whether hand position or hand posture (or both) can constitute a state of the action system that determines S-R compatibility. The present experiments represent an attempt to tease apart these variables.

In Experiment 1, we tested whether differences in response location alone (i.e., with a hand posture that remained identical-one that could be comfortably assumed at all locations) yielded different preferred pairings. In Experiment 2, we sought to demonstrate that such an effect does not obtain in a simple RT paradigm. In Experiment 3 , we tested whether differences in hand posture alone (i.e., with a location that remained the same) yielded different preferred pairings.

\section{EXPERIMENT 1}

In an attempt to determine whether location could by itself determine a preferred pairing, we sought a response in which the same hand posture could be employed at different positions along a frontoparallel line.

The response we used was a left or right deflection of the index finger. We believe that each response involves the use of one muscle, the posterior interosseus muscle, or its antagonist, the anterior interosseus muscle. The posterior interosseus muscle is used for abduction of the finger (i.e., movement toward the thumb); the anterior interosseus muscle, for adduction (movement away from the thumb; see Kapandji, 1970). ${ }^{1}$ The index finger was chosen because it also has "the greatest range of side-toside movement $\left(30^{\circ}\right)^{\prime \prime}$ (Kapandji, p. 158), making it the ideal response for testing the single-muscle hypothesis.

In sum, Experiment 1 was an attempt to determine whether effector position by itself is a sufficient condition for affecting the direction or size of the S-R compatibility effect.

\section{Method}

The reaction times of 12 right-handed Lake Forest College students were tested under each pairing rule at six positions. The response positions were 60,30 , and $0 \mathrm{~cm}$ to the left of the body midline for the left hand and 0,30 , and $60 \mathrm{~cm}$ to the right of the midline for the right hand, as illustrated in Figure 2 . The subjects activated one of two switches, to the right or to the left, in response to the appearance of a square either $3 \mathrm{~cm}$ above or $3 \mathrm{~cm}$ below a central fixation point on the monochrome monitor of an Apple IIe computer. The display arrangement was the same on all trials, and only the index finger was used; but the position of the switch apparatus and responding hand changed over six blocks of 80 trials (40 with a clockwise pairing rule and 40 with a counterclockwise rule).

As illustrated in Figure 2, the subject was seated at a table upon which sat the monitor and the switch apparatus. A black stripe on the table marked the center of the display, and the subject was instructed to keep the body (actually the "nose") aligned with the stripe. The switch apparatus was attached to a firmly seated block of wood ( $14 \mathrm{~cm}$ long $\times 4 \mathrm{~cm}$ high $\times 3.8 \mathrm{~cm}$ wide), which the subject grasped so that only the index finger moved in the simple sideto-side movement necessary to activate the switches (see the inset in Figure 2). To deflect the switch, the fingertip was moved approximately $1 \mathrm{~cm}$, and the "force" required was $15.5 \mathrm{~g}$. The distance of the right and left switches was approximately $1.2 \mathrm{~cm}$ from the center of the index finger position.

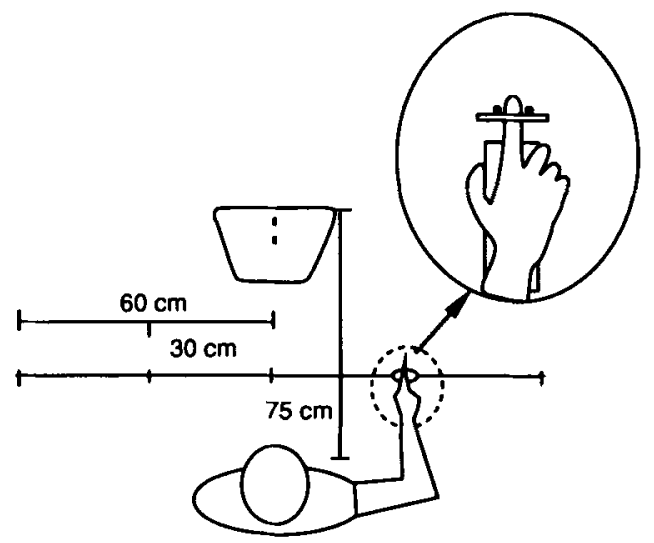

Figure 2. A top view of the arrangement for Experiment 1. Responses were solicited at five locations; the stimuli were presented on a centrally located computer monitor. 
The display of the fixation square was accompanied by a 100 msec warning beep. After $500 \mathrm{msec}$, one of the two squares appeared. The RT was measured to the nearest millisecond by an Applied Engineering Timemaster HO Clock Card in the computer. If the subject's response was correct, a 750-msec intertrial interval preceded the next warning beep. If the response was incorrect, it was followed by a 1-sec beep and a 2.5-sec time out. Trials on which errors occurred were repeated at the end of the block.

The independent variables (eccentricity, responding hand, and pairing rule) were balanced as follows. Six subjects performed the first half of the experiment with the left hand and the second half with the right; the other 6 were given the converse order. Eccentricity of response was balanced among subjects in blocks of 80 trials according to a Latin square design. The 80 test trials at each combination of hand and eccentricity comprised eight blocks of 10 trials (5 on which the top square was displayed and 5 on which the bottom square was displayed, in random order). Pairing rule was counterbalanced within these eight blocks (ABBAABBA). (The frequent change of pairing rule was intended to dissolve the longterm buildup of practice effects.) There were sets of 5 practice trials at each change in position and/or pairing rule. Practice blocks were repeated if the subject made an error.

\section{Results and Discussion}

The independent variables were hand, eccentricity $(0$, 30 , or $60 \mathrm{~cm}$ from the midline), S-R pairing rule (clockwise vs. counterclockwise), response direction (left or right), and blocks of trials. The median RTs of each block of five trials within condition was subjected to a five-way within-subjects analysis of variance (ANOVA).

The critical interaction is that between hand, S-R pairing, and eccentricity $[F(2,22)=9.68, p<.001]$; it is plotted in Figure 3. The eccentric positions show a lefthand preference for counterclockwise S-R pairing, whereas the right hand, conversely, shows a strong preference for clockwise pairings. At medial response positions, that preference diminishes and there is a suggestion that the converse pairing appears to emerge-at least for the left hand (though a Tukey post hoc test revealed this clockwise superiority to be nonsignificant). These preferences for $S-R$ pairings are the same as those found by Michaels (1989).

Two lower order significances are also captured in Figure 3. There was a significant main effect of eccentricity, with RTs faster at the medial position than at the lateral position $[F(2,22)=3.76, p<.05]$. The S-R pairing $\times$ hand interaction revealed that, averaged over eccentricity, the left hand responded faster with counterclockwise pairings than it did with clockwise pairings, whereas the right hand responded faster with clockwise pairings than it did with counterclockwise pairings $[F(1,11)=30.83, p<.001]$. This effect is opposite that observed by Bauer and Miller (1982), but consonant with that observed by Michaels (1989).

Though not bearing directly on the point of this experiment, response direction emerged as a significant factor in several effects. The means broken down by response direction are presented in Table 1. A main effect of response direction was observed, with responses to the right being faster overall than responses to the left $[F(1,11)$ $=7.74, p<.025]$. Eccentricity and response direction
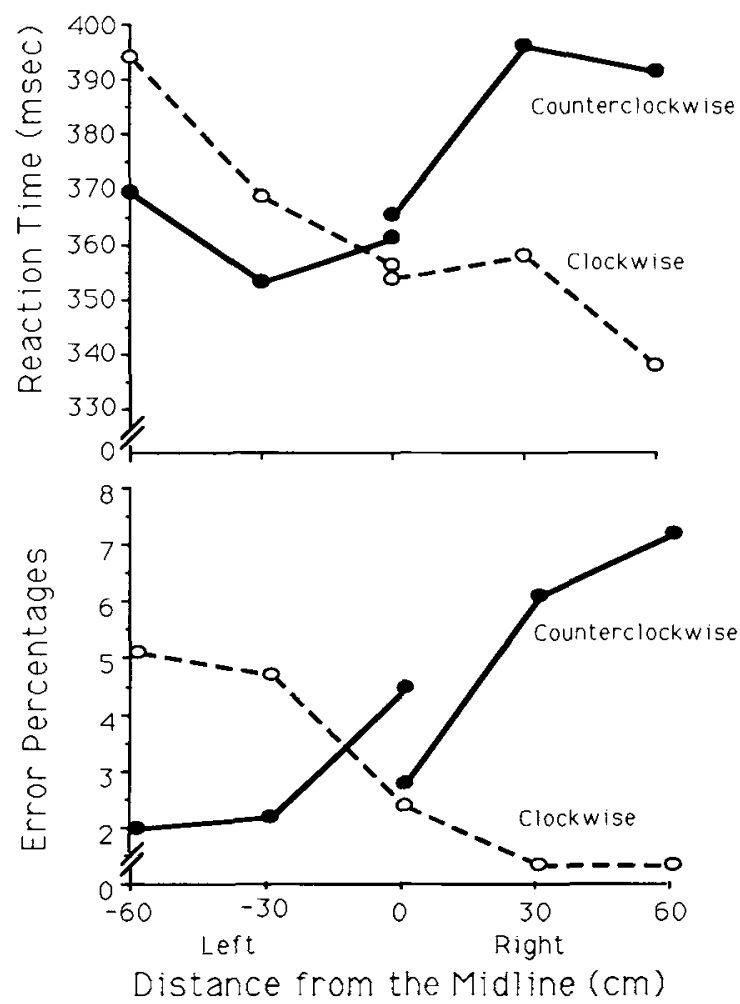

Figure 3. Average reaction times and error percentages as a function of stimulus-response (S-R) pairing, responding hand, and distance from the midline for Experiment 1 . The left and right hands appear to "prefer" opposite S-R pairings, with the preference abating as the medial position is approached.

interacted $[F(2,22)=4.60, p<.05]$, with left deflections being about the same at different positions but right deflections being faster on the midline. A second order interaction with hand, pairing, and response direction $[F(1,11)=7.55, p<.025]$ showed that the preference of the right hand for clockwise $S-R$ pairings over counterclockwise pairings was more dramatic for responses in the left direction than it was for responses in the right direction ( $47 \mathrm{vs.} 22 \mathrm{msec}$ ), whereas the left-hand response preferences for counterclockwise pairings over clockwise pairings were nearly equal $(13 \mathrm{msec}$ for responses in the left direction vs. $10 \mathrm{msec}$ for responses in the right direction). Response direction also interacted with eccentricity and hand $[F(2,22)=4.15, p<.05]$. One cell seemed to account for this; subjects were quite slow in making a right response with the left hand at the extreme position. This, along with the other direction effects, is likely to represent a problem of coordination, for the response is awkward for right-handed subjects; but since there was no third-order interaction of direction, hand, eccentricity, and pairing, the direction results do not weaken the conclusions drawn earlier.

Numbers of errors were collapsed over blocks of trials (to reduce the skew caused by the fact that most blocks had zero errors) and subjected to a four-way within-subjects 
Table 1

Median Reaction Times (in Milliseconds) Averaged over Blocks of Trials and Subjects in Experiment 1

\begin{tabular}{|c|c|c|c|c|c|c|}
\hline \multirow[b]{3}{*}{ S-R } & \multicolumn{6}{|c|}{ Distance from Midline } \\
\hline & \multicolumn{3}{|c|}{ Left-Hand Responses } & \multicolumn{3}{|c|}{ Right-Hand Responses } \\
\hline & $-60 \mathrm{~cm}$ & $-30 \mathrm{~cm}$ & $0 \mathrm{~cm}$ & $0 \mathrm{~cm}$ & $30 \mathrm{~cm}$ & $60 \mathrm{~cm}$ \\
\hline \multicolumn{7}{|c|}{ Counterclockwise Pairing } \\
\hline A-L & 361 & 368 & 376 & 383 & 406 & 405 \\
\hline B-R & 378 & 338 & 347 & 348 & 386 & 377 \\
\hline \multicolumn{7}{|c|}{ Clockwise Pairing } \\
\hline B-L & 399 & 382 & 365 & 359 & 355 & 341 \\
\hline A-R & 390 & 356 & 347 & 349 & 361 & 335 \\
\hline
\end{tabular}

Note- $A=$ above-fixation stimulus, $B=$ below-fixation stimulus, $\mathrm{L}$ $=$ left response, $\mathrm{R}=$ right response.

ANOVA. The results were similar to those for the RT scores, with slower RTs reflected in higher error rates (see the lower panel of Figure 3). The hand $\times$ S-R pairing $\times$ eccentricity interaction was again observed $[F(2,22)=$ $13.10, p<.001]$. Also significant was the hand $\times S-R$ pairing interaction $[F(1,11)=19.39, p<.005]$.

Both the error and the RT results, then, clearly demonstrated preferred S-R pairings for each hand, depending on position (as Michaels, 1989, had shown), even when hand posture was held constant. We concluded, therefore, that differential hand posture is not a necessary condition for altering the preferred pairing in an S-R compatibility of the sort reported by Bauer and Miller (1982).

\section{EXPERIMENT 2}

In Experiment 2, we sought to determine whether preferred pairings would emerge in a simple RT task. This was done to ensure that the pairings observed in Experiment 1 (and, by extension, in the Michaels, 1989, study) were not attributable solely to the characteristics of the motor system. This check was important, because Experiment 1 revealed some interactions involving response direction; we had to ensure that the eccentricity $\times$ pairing interactions were not merely an artifact of the ease of different responses.

Because it is not contingent upon the location of the stimulus, in simple RT the response can be organized prior to the appearance of the stimulus. Thus, we expected to find no compatibility effects (see, e.g., Callan, Klisz, \& Parsons, 1974). Experiment 2 was an explicit test of this, and, conversely, of just how powerful the above/below fixation stimulus might be in organizing a response. If position were to interact with S-R pairing, that would evidence a prepotency of stimulus position in organizing a response. If no such compatibilities should occur, it would imply that, with simple RT, responses are merely triggered by the stimulus and that any stimulus position is an equally effective trigger for any response position.

In Experiment 2, a simple RT task was employed; the stimuli and responses were the same as those in Experiment 1, and, as in Experiment 1, we tested all combina- tions of stimulus positions and response locations. The difference consisted of the absence of a stimulus position contingency.

\section{Method}

Twenty right-handed Lake Forest College students performed a simple RT task that was based on the task in Experiment 1.

The stimuli were exactly the same as those in Experiment 1, as was the apparatus. The only difference was that instead of being told to make a differential response (i.e., when the light appeared on the top, to press the switch to the left), the subjects were told to make the same response (e.g., to press the switch to the right) on a block of trials regardless of whether the light appeared above or below the fixation square. Each subject performed 80 trials in each response position; prior to every 10 trials, instructions appeared on the computer monitor, indicating response direction (i.e., "respond to the right").

\section{Results and Discussion}

Over 9,600 trials, there were only 26 errors, so errors were not analyzed further.

The median of each block of five RTs was subjected to a five-way within-subjects analysis of variance. Most important was that $S-R$ compatibility effects did not appear; S-R pairing rule was not a significant source of variance nor did it interact significantly with any other variable. The implications of this experiment for S-R compatibilities are cut and dried: Stimuli above and below fixation are equivalent triggers for left and right responses at all positions. The absence of $S-R$ compatibility in the simple case ensures that its observation in Experiment 1 cannot be understood solely with regard to effector mechanisms.

There was an interesting effect outside of the realm of compatibility. A significant first-order interaction was observed between hand and eccentricity $[F(2,38)=3.35$, $p<.05]$. As can be seen in Figure 4, the left hand was fastest in its most eccentric position, and it slowed as the medial position was approached; the converse was true for the right hand. For both hands, then, the further the response was to the right, the slower the response.

This observation is, incidentally, very different from other reports of response speed differences at different positions, which usually reveal an advantage for responses elicited in right hemispace, independent of which hand responds (e.g., Pierson-Savage \& Bradshaw, 1987). Given

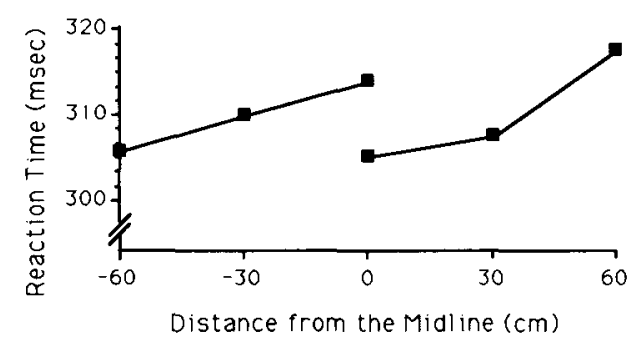

Figure 4. Experiment 2: Average reaction times as a function of responding hand and distance from the midline. 
that responding hand and hemispace are confounded in our experiment, our data add little to the understanding of Pierson-Savage and Bradshaw's hypothesized basis for hemispatial effects, the concept of functional processing space. We believe, nevertheless, that the notion of functional processing space might well benefit from an analysis of action-system variables of the sort explored in this series of experiments.

\section{EXPERIMENT 3}

Experiment 1 revealed that a hand placed in different positions but maintaining the same posture yielded different S-R compatibilities. This might imply that the effects reported by Michaels (1989) could be understood as positional rather than postural; but that hypothesis was deserving of a more rigorous test. Specifically, in Experiment 3, we sought to determine whether posture too, by itself, might affect $S-R$ compatibility.

There are already suggestions in the literature that postural manipulations can affect perception. Oldfield and Phillips (1983), for example, showed that tactile form perception can depend on posture. More to the point, postural effects have also been shown in S-R compatibility. In particular, the relation of compatibility to posture has been examined in connection with the thesis that there are anatomically left and right fingers, which presumes a prone (palm-down) posture (Ehrenstein, Schroeder-Heister, \& Heister, 1989; Heister, Ehrenstein, \& SchroederHeister, 1986). These authors try to separate strict spatial compatibility effects from anatomical compatibility effects. Heister, et al. (1986) used prone and supine (palmup) hand postures to dissociate spatial left/right fingers from anatomical left/right fingers when stimulus and response directions were parallel. Ehrenstein et al. (1989) used orthogonal directions (left/right stimuli and near/far responses [made with anatomically left or right fingers]). Their relevant conditions ( $B$ and $C$ ) revealed that although posture did not reverse the compatibility, it did influence the size of its effect. Their results, although couched in the language of spatioanatomical mappings, translate into the clockwise/counterclockwise terminology as follows: The left hand preferred clockwise pairings and the right, counterclockwise, in both prone and supine postures; but the latter preference was bigger with the prone posture (Ehrenstein et al., 1989).

The goal in Experiment 3, then, was twofold. First, it was intended to replicate the Ehrenstein et al. (1989) observation that posture, by itself-with position held constant-might affect the size or direction of the preferred pairing. Second, it was meant to test whether the use of anatomically left and right effectors is a necessary condition for the appearance of postural effects. If the response is not made by anatomically left and right fingers, then any differential compatibility effects due to posture will not be due to different spatioanatomical mappings.

\section{Method}

Eight right-handed Lake Forest College students made left/right deflections of a centrally located toggle switch, held between the thumb and forefinger, to squares above or below fixation on a computer screen. There were eight blocks of 60 trials ( 10 practice trials and 50 test trials). On half of the blocks, the subjects responded with the left hand; on half of the blocks, they responded with the right hand. On half of the hand blocks, the subject's hand was lightly restrained into a prone posture (palm down), and on half it was lightly restrained into a supine posture (palm up). On one such handposture block, the subjects were instructed to deflect the switch to the left for a top square and to the right for a bottom square-a counterclockwise pairing-and on another block, the subjects were instructed to use the converse pairing. Posture was balanced between subjects, half starting with four blocks of supine responses and half starting with four blocks of prone responses. Hand and pairing rule were counterbalanced within the four blocks at each posture. The display and switch were centered on the body midline (i.e., the center position in Figure 2). In all other respects, Experiment 3 was the same as Experiment 1.

\section{Results and Discussion}

A five-factor within-subjects ANOVA was performed on the median RTs from each block of 5 trials. The independent variables were posture, hand, pairing rule, response direction, and blocks of trials. Only one significant $F$ was found, the critical one: the posture $\times$ hand $\times$ pairing rule interaction $[F(1,7)=5.99, p<.05]$. This interaction is plotted in Figure 5. (The results, broken down by response direction, are presented in Table 2.)

Figure 5 reveals that the preferred pairing depends on posture as follows. When the subject is responding with the left hand in the prone position, the preferred pairing is clockwise, as reported by Bauer and Miller (1982). If, alternatively, responses are made from a supine posture, then the counterclockwise pairing rule leads to shorter RTs. Similar but less striking results occur for the right hand: Bauer and Miller's preferred relation appears to hold for prone postures, but the converse is suggested for supine postures.

A similar analysis was performed for the error data, and, as in Experiment 1, we collapsed errors over blocks because the errors were so few. This analysis also revealed only one significant effect, the two-way interaction of posture and hand $[F(1,7)=9.33, p<.025]$. As is shown in Figure 5, the left hand made more errors in the prone posture when the right hand tended to make more errors while held supine. Although the error data do not demonstrate pairing-rule differences, they make clear that the RT results are not due to speed-accuracy tradeoffs.

The statistical significance of the results of Experiment 3 is not high, but when these results are considered together with the effects reported by Heister et al. (1986) and Ehrenstein et al. (1989), it seems clear that effector posture, as well as effector position, can affect S-R compatibility. In addition, since the responses were always made with the same pairs of digits (the thumbs and forefingers), there is no appeal to spatioanatomical mapping (Heister et al., 1986) as a possible basis for the compatibility. 


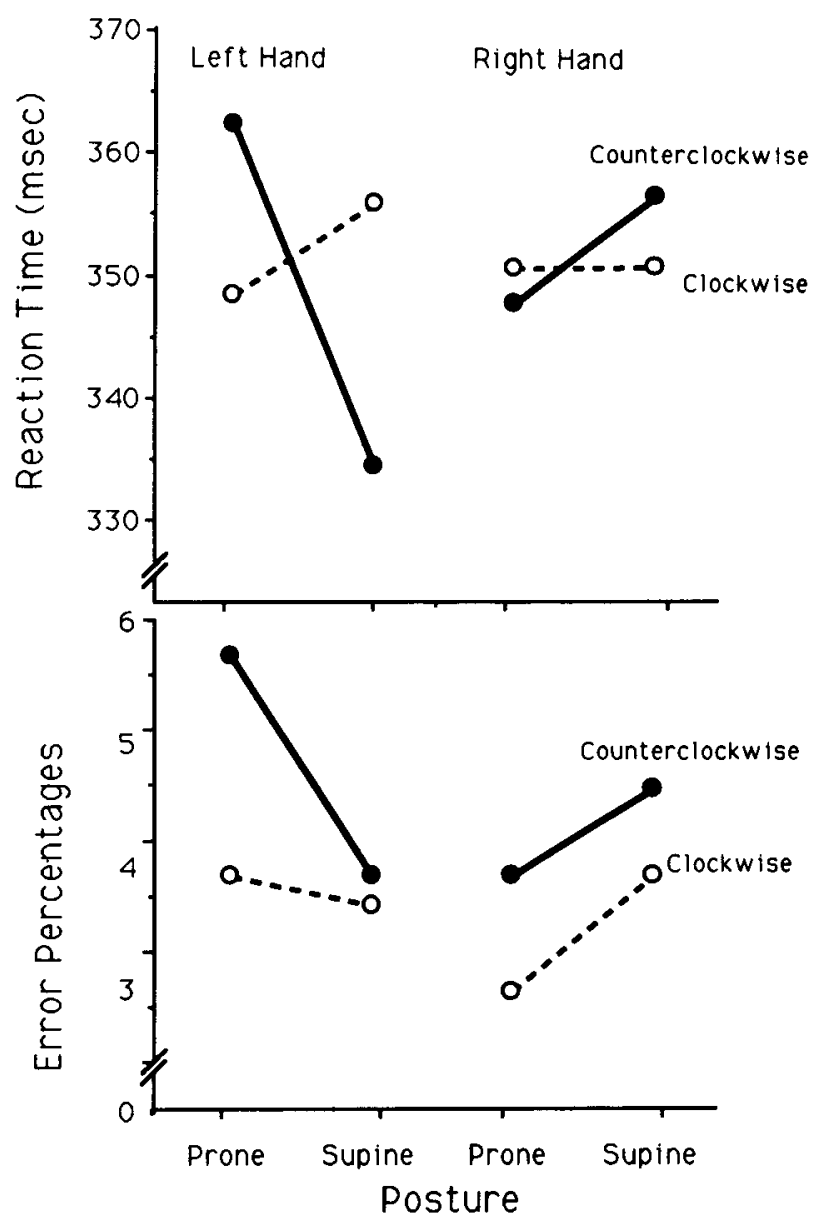

Figure 5. Average reaction times and error percentages as a function of responding hand, pairing rule, and hand posture for Experiment 3.

\section{GENERAL DISCUSSION}

The point of departure for the present series of experiments was the idea that action sets up perception-that not only the structure of the motor system, but also its current organizational state, can condition perception. The dependence of perception on the state of the action system was offered as a possible basis for S-R compatibility effects in RT. For example, the intention to point at or to intercept an apparently moving square can yield different compatibilities for the same combinations of stimuli and responses (Michaels, 1988). Similarly, Michaels (1989) claimed that the alteration of even lower level motor system states can affect S-R compatibility relationsfor example, that the "same response" will reveal different S-R compatibilities, depending on local details of the motor system, such as the hand that is doing the responding (Bauer \& Miller, 1982) or the manner of that response (a combination of location and posture; Michaels, 1989).

Within this theoretical framework, the experiments reported here were intended particularly to determine whether the motor system variables of hand posture and hand position could each, by themselves, effect a change in the direction or magnitude of S-R compatibilities. Both variables illustrated such an effect. ${ }^{2}$

Experiment 1 demonstrated the effectiveness of effector position in influencing S-R compatibility, and Experiment 3 demonstrated the importance of hand posture. We should note, of course, that both experiments could be understood in terms of postural changes; Experiment 1 did not exclude the possible role of body posture (e.g., limb position, head position, trunk position). Obviously posture and position cannot be completely decoupled, since positional changes, of necessity, implicate postural changes. The best we can do is to decouple them locally (e.g., at the hand), as was done in Experiment 1.

One rather surprising feature of these compatibility effects is that very "low-level" stimuli are sufficient to obtain the effect. Usually in ecologically motivated experiments on perception and action, the stimuli are complex patterns rich in kinematics. In spite of their apparent simplicity, the information provided by a square above or below fixation was sufficient to reveal the interrelation with the action system. The role played by these actionsystem variables made itself known when involved, again, in a surprisingly simple task (left or right finger deflections or switch deflections), in comparison with, say, a task such as baseball batting.

To argue simply that action-system states affect S-R compatibility, as we have done thus far, is, we confess, more of a skeleton of a theory of $S-R$ compatibility than a theory of S-R compatibility. We will conclude, therefore, by considering how the current results bear upon our admittedly sketchy theory and the family of theories that we hope to displace.

The traditional approach to the relation of perception and action, in general, and to S-R compatibility, in particular, separates the detection of information from the organization of action. Integrating them-to map the sensory onto the motor-is conceived to be a job for the perceiver, in which different sensorimotor mappings are thought to differ in difficulty. The goal of the traditional research, then, is to establish (1) what the relevant stimulus dimensions are, (2) what the relevant response dimensions are, and (3) how one integrates them-that is, what

Table 2

Median Reaction Times (in Milliseconds) Averaged over Blocks of Trials and Subjects in Experiment 3

\begin{tabular}{|c|c|c|c|c|}
\hline \multirow[b]{3}{*}{ Pairing Rule } & \multicolumn{4}{|c|}{ Response Direction } \\
\hline & \multicolumn{2}{|c|}{ Left Hand } & \multicolumn{2}{|c|}{ Right Hand } \\
\hline & Left & Right & Left & Right \\
\hline \multicolumn{5}{|c|}{ Prone } \\
\hline Counterclockwise & $371^{*}$ & 352 & $354^{*}$ & 340 \\
\hline Clockwise & 350 & $345^{*}$ & 346 & $354^{*}$ \\
\hline \multicolumn{5}{|c|}{ Supine } \\
\hline $\begin{array}{l}\text { Counterclockwise } \\
\text { Clockwise }\end{array}$ & $\begin{array}{l}341^{*} \\
351\end{array}$ & $\begin{array}{l}326 \\
360^{*}\end{array}$ & $\begin{array}{l}364^{*} \\
349\end{array}$ & $\begin{array}{l}347 \\
352 *\end{array}$ \\
\hline
\end{tabular}

*Responses given to the stimulus above fixation. 
rules inside the perceiver tie the stimulus dimensions to the response dimensions?

The ecological approach to perception and action forcefully argues against the separation of stimulus and response dimensions. Instead, the dimensions of information are taken to be the dimensions of action, and their separation is conceived to be a complicating fiction. One point in the current study, as in the preceding one (Michaels, 1989), has been to illustrate just how complicated that fiction can become-to show that, from a traditional perspective, a perceiver must take into account the response dimensions of hand, location, and posture, among other things, in effecting a mapping.

The ecological approach asks how the activity of the muscles might be directly modulated by information. An elaboration of such a theory (see Turvey, 1990, for a recent review) and a more formalized ecological approach to choice RT must await that elaboration. The expectation is that an understanding of the nature of coordination will provide the backdrop for identifying the nature of information that can regulate it. The choice RT paradigm-with its typically simple stimuli and responsesoffers only a Maxwellian peephole view of the rich landscape of motor coordination with respect to vision.

As to which approach to understanding S-R compatibility - the traditional or the ecological-is better, our tack has been to claim that the increasing number of apparently relevant response dimensions puts an increasingly cumbersome burden on the notion of sensorimotor transformation. The increasing number of independent contingencies will, we believe, make the theory that perceivers must integrate stimulus and response dimensions fall from its own weight.

One could argue, of course, that the concept of sensorimotor integration is still good, and that our common problem is that science has not yet correctly identified the dimensions of stimuli and responses that are integrated. For example, perhaps the contingencies that we have highlighted are not independent, and an appropriately complex or abstract conceptualization of the stimulus might indeed map simply and naturally onto the appropriately abstract or complex conceptualization of what it means to coordinate the action. But that, of course, is our point: The characterization of the stimulus must fit naturally together with the characterization of the action. When it does, notions of sensorimotor integration or transformation as mediating processes would become superfluous.

\section{REFERENCES}

Bauer, D. W., \& Miller, J. (1982). Stimulus-response compatibility and the motor system. Quarterly Journal of Experimental Psychology, 34A, 367-380.

Callan, J., Klisz, D., \& Parsons, O. A. (1974). Strength of auditory stimulus-response compatibility as a function of task complexity. Journal of Experimental Psychology, 102, 1039-1045.

FITch, H., \& TURVEY, M.T. (1978). On the control of activity: Some remarks from an ecological point of view. In $D$. Landers \& $R$. Christina (Eds.), Psychology of motor behavior and sport (pp. 3-35). Urbana, IL: Human Kinetics.

Ehrenstein, W. H., Schroeder-Heister, P., \& Heister, G. (1989). Spatial S-R compatibility with orthogonal stimulus-response relationship. Perception \& Psychophysics, 45, 215-220.

Heister, G., Ehrenstein, W., \& Schroeder-Heister, P. (1986). Spatial S-R compatibility effects with unimanual two-finger choice reactions for prone and supine hand positions. Perception \& Psychophysics, 40, 271-278.

Hubbard, A. W., \& Seng, C. N. (1954). Visual movements of batters. Research Quarterly, 25, 42-57.

KAPANDI, I. A. (1970). The physiology of the joints (Vol. 1). New York: Churchill Livingstone.

MiChaELS, C. F. (1988). S-R compatibility between response position and destination of apparent motion: Evidence of the detection of affordances. Journal of Experimental Psychology: Human Perception \& Performance, 14, 231-240.

MiChaELS, C. F. (1989). S-R compatibilities depend on eccentricity of responding hand. Quarterly Journal of Experimental Psychology, 41A, 263-272.

Oldfield, S. R., \& Phillips, J. R. (1983). The spatial characteristics of tactile form perception. Perception, 12, 615-626.

Pierson-Savage, J. M., \& Bradshaw, J. L. (1987). Mapping of extracorporeal space by vibrotactile reaction times: A far-left-side disadvantage. Perception, 16, 283-290.

Turvey, M. T. (1990). Coordination. American Psychologist, 45 , 938-953.

WARREN, W. (1984). Perceiving affordances: Visual guidance of stair climbing. Joumal of Experimental Psychology: Human Perception \& Performance, 10, 683-703.

\section{NOTES}

1. We thank Karl Newell for suggesting this response.

2. In an additional experiment, postural and positional effects were contrasted, but transfer effects over blocks of trials made the results too complex to interpret. The modulation of $S-R$ compatibilities by such transfer effects are, incidentally, probably responsible for the differences between the magnitudes of differences between pairings at the medial positions in the Bauer and Miller (1982) study (in which only medial responses were used) and in the Michaels (1989) experiment (in which both medial and eccentric locations were used). The presence of eccentric conditions moderated the preferred pairings at the medial position.

(Manuscript received April 9, 1990; revision accepted for publication November 28,1990 .) 